\title{
Clinical criteria for the successful curing of composite materials
}

\author{
Mahn $\mathrm{E}^{1}$
}

\begin{abstract}
As composites have continued to be optimized, significant differences in physical, mechanical, and clinica performances between the available systems have lessened. Yet, despite all the improvements, one constant remains: direct composites need to be light-polymerized. Clinicians need to understand the principles of light-curing because unbound monomers are cytotoxic and improperly cured composites are less biocompatible. Presently, there are four technologies available to cure composites. Once the light source is chosen, the clinician should consider several factors to ensure that the composite is being cured satisfactorily. This article analyzes the various current technologies, their strengths and weaknesses, and the relevance of following certain protocols to ensure proper polymerization rates.
\end{abstract}

Rev. Clin. Periodoncia Implantol. Rehabil. Oral Vol. 6(3); 148-153, 2013.

Key words: History, curing depth, light intensity, different methods.

\section{INTRODUCTION}

The field of application of the first dental composite materials was very limited compared to that of amalgam. Early composite materials were suited only for anterior restorations because of their weak mechanical properties. In the past few decades, however, dental composites have developed from an inferior resin material into the material of choice for the fabrication of highly esthetic, durable posterior and anterior restorations in direct restorative dentistry. The developments in the field of composite technology resulted in the advent of new classes of materials and fields of application, such as adhesive luting materials, resin-modified glass ionomers, core buildup materials, ormocers and compomers. Working with resin-based materials requires a detailed understanding of the curing properties and combination possibilities of these materials and the factors that affect this process. Most modern composite materials are either based on a light-curing technology or offer a dual mechanism of light- and selfcuring technology. In the case of light-curing materials the clinician need to assure that enough photons reach the bottom of the composite, or the composite will not achieve the properties necessary for long term survival, as seen in Figure 1.

\section{A BRIEF HISTORY OF COMPOSITES}

Self-curing composites dominated the field of tooth-coloured dental restorations for many years before light-curing materials captured the market. These self-curing composites were based on radical polymerization initiated by the decomposition of benzoyl peroxide. These materials were usually supplied in two-component systems, usually consisting of an activator in an amine-containing base paste and an initiator in a peroxide-containing catalyst paste. A drawback of the chemical curing process is that users have to wait for a relatively long time after they have applied the materials before they can continue with the treatment. Final polishing can be performed after 24 hours at the earliest. Furthermore, these composites are not stable at room temperature and therefore require refrigerated storage below $8^{\circ} \mathrm{C}$. These difficulties can be avoided by using photo-polymerization. An ultraviolet light-activated fissure sealant was one of the first lightcuring materials that became available ${ }^{(1)}$. However, the ultraviolet light-cured systems were soon replaced because they involved some serious drawbacks, such as the warm-up period of the apparatus, a limited depth of cure of 1 to 2 millimetres and UV radiation exposure, which may cause damage to the corneal epithelium. The next stage was to introduce visible light-activated composites. To enable the formation of organic-chemical compounds, the photoinitiator campherquinone was most commonly used in these materials ${ }^{(2,3)}$.

\section{A SHORT HISTORICAL SURVEY OF CURING LIGHTS}

The wide array of curing lights available on the market may be at times confusing. A review of the characteristics of these curing lights may help to highlight their strengths and weaknesses:

\section{Halogen Lights}

Basically, halogen lights are based on the same technology as conventional light bulbs with the difference that the temperature of the filament is several hundred degrees higher. The light of a tungsten halogen curing light is produced when the thin tungsten filament, which works as a resistor, is heated by the current to temperatures of around $3000^{\circ}$ Kelvin. When the filament becomes incandescent, it emits electromagnetic radiation in the form of visible light as well as a large amount of infrared radiation. As high temperatures are produced in the process, heat elimination is indispensable. Furthermore, the lifetime of the halogen bulb is limited (enabling approx. 6 months of clinical use). Halogen lights are reliable and cure all composite materials within a reasonable period of time. They can be purchased at relatively low acquisition costs and burned-out bulbs are relatively inexpensive to replace.

\section{Plasma Arc Curing Lights (PAC)}

These lights were introduced to the dental profession with the claim that they would minimize curing times without affecting the mechanical properties of the cured materials. Practitioners were astonished by how quickly these lights cured restorative composites. On average, the recommended exposure time to cure an increment of a light-coloured composite was between 3 and 5 seconds. In addition to their high prices, one of the problems encountered in these lights is that a number of composites, adhesives and protective varnishes do not cure with high-intensity light sources such as PAC lights and argon lasers, because of an incompatibility between the photoinitiator of these

1. $D D S, D M D, P h D$. Universidad de los Andes. Chile. 
materials and the light emission wavelength. Even if the photoinitiator is compatible with the wavelength range of the emitted light, a few studies have shown that the short curing times recommended by the manufacturers are insufficient for many composite materials ${ }^{(4,5,6)}$.

\section{Light Emitting Diode (LED) Lights}

The use of LED technology to polymerize dental materials was mentioned more than ten years ago ${ }^{(7)}$. The main advantages of this technology include moderate costs, the improved light-intensity of second-generation LED lights (with a corresponding reduction of the curing time), practical features (e.g. smaller, more portable and cordless lights) and the long service life of diodes (approx. 1000 hours, which is equivalent to 5 years of clinical use approximately). A number of studies have shown that LED lights are able to polymerize composite materials to a depth of cure $^{(8)}$, compressive strength ${ }^{(9)}$ and flexural strength ${ }^{(10,11)}$ that are statistically not different from the values obtained in composite materials cured with conventional halogen lights using the same light intensity ${ }^{(12)}$

Furthermore, Mills et al. ${ }^{(13)}$ found in a study that the LED lights produced a statistically significant higher depth of cure in three differently filled, medium-shade composite materials than did the halogen units when comparable curing times were used. The first and second generation of LED lights were not able to cure some materials because of incompatible wavelength ranges (similar to PAC lights). Some manufacturers added with the polywave ${ }^{\circledR}$-LED an emission peak at $405-410 \mathrm{~nm}^{(14)}$ in the wavelength range of the third and latest generation of LED lights. As the cost for LED lights continues to decrease (they cost about the same as halogen lights but the life of their light source is longer) and as they offer a high degree of portability (battery operation), high light intensity and a wavelength range that is similar to that of halogen lights, the future of LED curing lights looks promising, fact that is shown in the wide range of products offered to the dental practitioner.

\section{Curing Depth, Light Intensity and Polymerization Time}

Determining whether or not a composite restoration is completely cured is one of the largest challenges in the clinical practice. The uppermost layer is cured almost immediately while the deeper areas are still reacting. Practitioners have to be aware of the fact that the energy is attenuated and dispersed with increasing material depth. There is therefore a risk that the monomer may not convert into a polymer.

Several factors affect the depth of cure of composite materials, with shade and translucency being the most significant ones. For example, the curing depth is lower if a dark and opaque composite is polymerized ${ }^{(15)}$. The light can penetrate more deeply with a light or translucent shade than with an opaque material. The same effect is achieved when shade A1 is compared with shade A3.5 or A4 (Figure 2).

\section{The Composite Structure Also Affects Light Penetration}

In particular, the very small particles $(0.04 \mathrm{~mm})$ of microfilled composites scatter the light, which decreases light penetration (comparable to the effect of fog on the headlights of cars) ${ }^{(16)}$. The same effect occurs in hybrid composites, which contain microfine particles. These materials impede light penetration to a greater extent than do composites that are free of microfillers ${ }^{(16)}$.

Unfortunately, many practitioners are not aware of this limitation in the light-induced polymerization process of composites. German researchers published data which showed that $44 \%$ of the users change the polymerization time when they use a different material and only $14 \%$ of them know the relevance of the composite shade to the curing process. This indicates an urgent need for information, as the shade and translucency play a significant role in the curing process ${ }^{(16,17)}$.

The polymerization depth (D) is generally expressed by the formula $D=c^{1} \cdot \log \left(c^{2} \cdot L \cdot T\right)$, The factors $c^{1}$ and $c^{2}$ contain data regarding the concentration of initiators and the absorption coefficients of the composite. A number of researchers were able to prove the logarithmic relationship between the curing depth and the light intensity as well as between the curing depth and exposure time shown in this formula ${ }^{(18,19)}$. For the clinical practice, this formula shows that in cases in which the light intensity is increased by a certain factor, the exposure time can be decreased by the same factor.

It would be ideal for dental practitioners to be able to take advantage of a curing protocol that can be applied universally in all cases. Koran and Kürschner ${ }^{(20)}$ concluded that if the total dose of light intensity (the product of light intensity and exposure time) delivered to the composite is higher than $17.000 \mathrm{mWs} / \mathrm{cm}^{2}$, the overall surface hardness tends to remain constant. This dose is achieved with a light intensity of $400 \mathrm{~mW} / \mathrm{cm}^{2}$ and an exposure time of 42.5 seconds $\left(17.000 \mathrm{mWs} / \mathrm{cm}^{2}\right.$ : $400 \mathrm{~mW} / \mathrm{cm}^{2}=42.5 \mathrm{sec}$ ), or, to mention an example, an exposure time of 20 seconds requires a light intensity of $850 \mathrm{~mW} / \mathrm{cm}^{2}$. This calculation is referred to as the "Total Energy Concept".

Studies concluded that a light intensity of at least $300 \mathrm{~mW} /$ $\mathrm{cm}^{2}$ is required ${ }^{(21,22,23)}$ to cure 1 millimetre of material thickness. On the other side, independent from the intensity, it is generally not possible to sufficiently cure increments that are thicker than 2 millimetres $^{(23)}$.

It is important to point out that these tests were performed in laboratories under ideal conditions; an additional safety margin should be included for applications in the dental practice. It is therefore not advisable to use light intensities below $400 \mathrm{~mW} / \mathrm{cm}^{2}$. In addition, practitioners should take into account that the light output of the light guide may be hampered by disposable infection control barriers ${ }^{(24)}$, debris, contamination, composite residue ${ }^{(25)}$, scratch marks, discoloration and damage ${ }^{(26,27)}$.

\section{THE BLEACH DILEMMA}

Most light-curing dental composites use campherquinone as the initiator for light-induced polymerization. Campherquinone offers a broad absorption spectrum (400-500 nm) with peak absorption at 465$470 \mathrm{~nm}$. It is a solid yellow powder with an unbleachable chromophore group (Figure 3). Composite materials exhibit an undesirable yellowing effect, even after polymerization with medium amounts of campherquinone.

To attenuate this effect, some manufacturers have begun to add other photoinitiators, which work alone or synergistically with camphorquinone ${ }^{(28)}$. Acylphosphine oxides are often utilized for this purpose because of their weak yellowish-whitish tinge. The absorption peak of these compounds is located predominantly in the UV region with a small extent in the visible light region (violet light).

Second-generation LED curing units, which have a peak at 468 $\mathrm{nm}^{(29)}$ and perfectly match the absorption peak of campherquinone, are ineffective in conjunction with these new initiators. This problem does not occur with halogen lights, because they offer a broad emission spectrum. Neumann et al. ${ }^{(30)}$, investigated the performance of two LED units and a halogen light in conjunction with campherquinone-containing and non-campherquinonecontaining formulations. This investigation highlighted the difference in the effectiveness of these curing lights and the need for an overlap between the emitted light and the absorption spectrum of the initiators.

The use and curing of initiators such as trimethylbenzoyldiphenylphosphine oxide (TPO) (Lucirin TPO®, Figure 3), phenylpropandione (PPD) and bisacylphosphine oxide (Irgacure 819 ) is particularly problematic in translucent and bleach-coloured composites and in conjunction with LED units of the first and second generation. In these materials, the yellow initiator (campherquinone) is used in a reduced concentration or completely eliminated. Lucirin TPO ${ }^{\circledR}$ is the most popular of the above mentioned "new" initiators. This ingredient is utilized for low-yellowing, white pigmented UV-curable coatings, printing inks and clearcoats in the industry ${ }^{(31)}$. In chemical terms, this compound is a 2, 4, 6-trimethylbenzoyldiphenylphosphine oxide $^{(31)}$. Although the absorption peak of Lucirin TPO $®$ is located

in the UV spectrum, it is sensitive to visible light. Because of this characteristic, it is suitable for dental applications. However, it does not cure with all light-curing units.

In the past few years, new LED curing units offering a broadband emission spectrum were launched. As a result, these lights 
are theoretically suitable for curing all dental materials, including those composites which contain whitish initiators such as Lucirin TPO®. Ultralume ${ }^{\circledR}$ (Ultradent, South Jordan, Utah, USA) and G-light ${ }^{8}$ (GC, Tokio, Japan) were pioneers of this category of polymerization units. Valo ${ }$ (Ultradent, South Jordan, Utah, USA), Smartlite ${ }^{\circledR}$ Max (Densply DeTrey, Konstanz, Germany) and bluephase ${ }^{\circledR}$ G2 and bluephase Style ${ }^{\circledR}$ (Ivoclar Vivadent, Schaan, Liechtenstein) are among the latest LED lights that have become available on the market and offer this characteristic. The spectral output of these units is unique because they incorporate a standard peak at $465-470 \mathrm{~nm}$ and a secondary peak in the range of $404 \mathrm{~nm}^{(14)}$, with considerable variation between the various units investigated. Figure 4 shows the light emission spectrum of different curing units. Price et al. ${ }^{(14)}$, tested the new broadband spectrum of the LEDs by comparing two LEDs with a broadband spectrum with two standard LED lights. The highest overall hardness was, in fact, achieved by the units that use the new polywave $\AA$ technology. In a recent study, Giorgi et al. ${ }^{(32)}$, also found differences in the degree of cure between composites cured with $2^{\text {nd }}$ and $3^{\text {rd }}$ generation LEDs, with better results for 3 rd generations LEDs in combination with more translucent composites due to the overlap of emission spectrum with emission absorbtion of the composite resin ${ }^{(32)}$. Also recently, Santini et al. ${ }^{(33)}$, proved that the use of polywave LEDs significantly improves both DC (degree of conversion) and $\mathrm{KHN}$ (Knoop microhardness) of materials containing TPO.

\section{POLYMERIZATION TECHNIQUES}

Several studies were carried out and numerous techniques invented in an effort to find the best curing method. Probably the most sophisticated technique was developed by Lutz et al. ${ }^{(34)}$, in 1986 . This technique uses a light-reflecting wedge at the cervical margin and transdental polymerization at the axial walls. This technique was found to be effective not only by the developer but also by other clinical investigators ${ }^{(35,36)}$. It should be noticed, however, that a substantial decrease in light intensity occurs in conjunction with this technique; Goes et al. ${ }^{(35)}$, found that only $8 \%$ of the light was transmitted through the reflecting wedge.

Several years later, studies showed that the effectiveness of this technique was attributable to the low polymerization stress, which results from the reduction in light intensity during transdental curing ${ }^{(36)}$, rather than to the re-direction of light with a light-reflecting wedge. In fact, transdental polymerization resulted in a decrease in the light intensity and this affects the properties of the material ${ }^{(37)}$

A few years later, Versluis et al. ${ }^{(38)}$, showed that composites do not shrink towards the light. The literature on curing techniques does not provide a clear indication that any of these methods produces better results than straightforward direct light curing.

The loss of energy in relation to an increase in distance is another essential factor that has to be taken into account. Ideally, the tip of the light guide should be in direct contact with the restoration. However, this is not always possible in clinical applications; distances of more than $8 \mathrm{~mm}$ between the light emission window and cavity floor were demonstrated in proximal restorations ${ }^{(39)}$.

There is no doubt about the fact that the light intensity decreases with an increase in distance. However, there is no consensus in the literature regarding the amount of energy that is lost. Pires et al. ${ }^{(40)}$, reported a decrease of $22 \%$ at a distance of $2 \mathrm{~mm}$ and $53 \%$ at $6 \mathrm{~mm}$. Prati et al. ${ }^{(41)}$, observed a decrease of $39 \%$ at $2 \mathrm{~mm}$ and $77 \%$ at $6 \mathrm{~mm}$. However, the distances used in these studies hardly bear any relevance with the clinical reality. The distance between the tip of the light guide and the cavity floor of a typical class II preparation was reported by Price et al. ${ }^{(42)}$, to be $6.3 \mathrm{~mm}$ (standard deviation $+/-0.7 \mathrm{~mm}$ ).

In an approach that reflected real-life conditions more appropriately, Meyer et al. ${ }^{(11)}$, observed a decrease between $33 \%$ and $83 \%$ at $10 \mathrm{~mm}$, depending on the curing light and the light guide in particular. These results were confirmed in another study carried out by Price et al. ${ }^{(43)}$, they observed significant differences among the curing lights and considerable decreases in the light intensity, especially when turbo light guides were used. In the same study, the same curing light (Optilux 501, SDS Kerr Demetron, Danbury, CT, USA) was tested with a parallel-wall and a turbo light guide: the decrease in light intensity was $33 \%$ and $44 \%$, respectively.

Furthermore, restorations in the posterior region in particular are difficult to reach, which may have a further adverse effect on the polymerization process. Dental assistants and even dentists tend to underestimate the importance of the distance between the light emission window and composite restoration. It is therefore advisable to use a parallel light guide, if the curing unit produces sufficient energy, to ensure an appropriate polymerization process.

Another important factor is the behaviour of the LED when it is used without a light probe. In absence of a light probe, severe divergence of light occurs and the loss of energy dramatically increases at a distance of a few millimetres ${ }^{(43)}$. Basically, the problem consists in the nature of LEDs, because they emit light in $180^{\circ}$ (Figure 5). This considerable loss of energy was confirmed by Price et al. ${ }^{(43)}$, when they used the curing lights of various manufacturers without light guides. A graphic example of this phenomenon can be seen in Figure 6 . Curing lights that are used without light guides are unsuitable for dental applications because of the dramatic loss of light intensity.

Damage or contamination of the light guide may also cause a decrease in the intensity of the curing light. Ernst et al. ${ }^{(25)}$, found an increase in intensity of $38 \%$ after the light guides had been cleaned and $38 \%$ of the contaminated light guides had never been removed before.

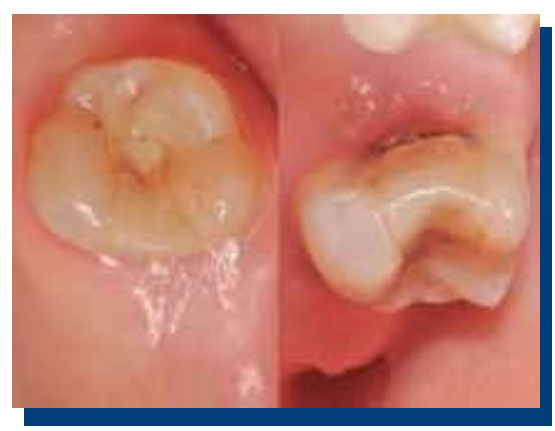

Figure 1. Secondary caries due to insufficient polymerization of the composite? If insufficient light reach the bottom of the composite, caries adjacent to restorations (CAR) can occur.



Figure 2. Depth of cure of a micro-hybrid composite, depending from the shade and translucency. 


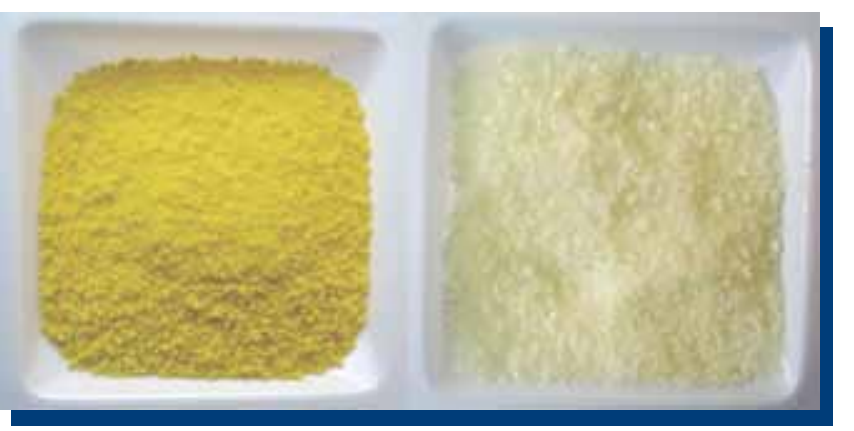

Figure 3. Campherquinone (left) and Lucirin TPO® (right).

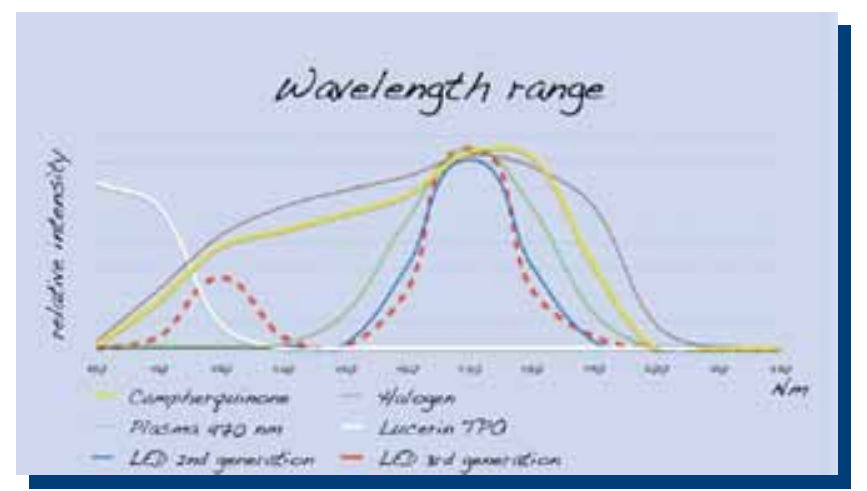

Figure 4. Emission spectra of various curing units and absorption spectra of various photoinitiators.

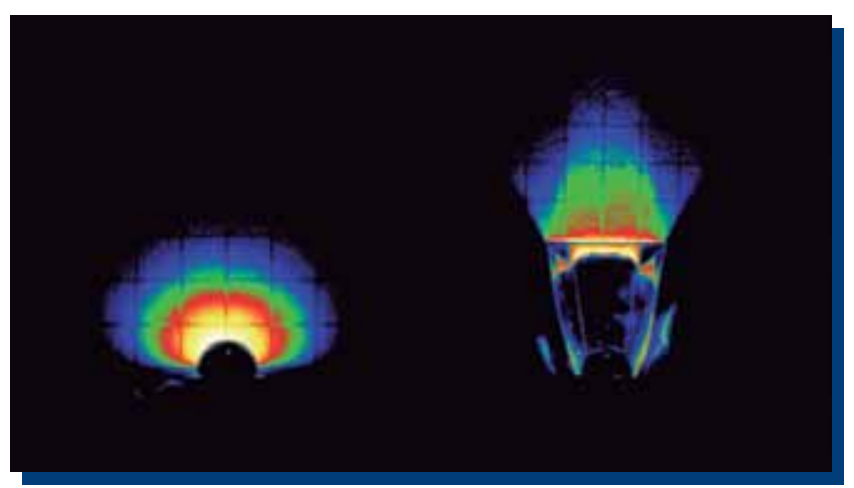

$\overline{\text { Figure 5. Light divergence in an LED without a light guide (left) and focused light }}$ with a light guide (right).

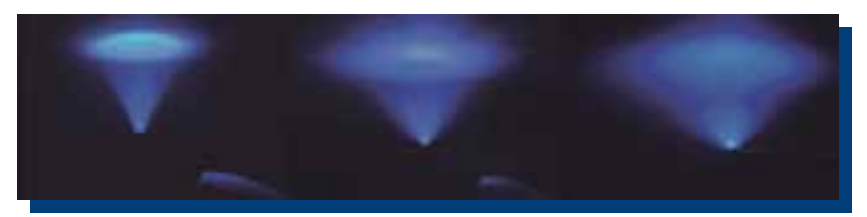

Figure 6. Divergence with a parallel light guide (left), with a turbo light guide (center) and without a light guide (right).

\section{SOFT START, PULSE DELAY, RAMP CURE: INTERMITTENT OR CONTINUOUS CURING?}

The literature includes numerous studies that appear to show that modified curing methods have a beneficial effect on the polymerization of direct composite restorations ${ }^{(44,45)}$. These methods are based on the understanding that a slower conversion rate enhances the flow of the material, which reduces the shrinkage stress of the composite and therefore helps prevent gap formation ${ }^{(20,46)}$.

However, these methods should not affect the degree of cure and the surface hardness to ensure the mechanical properties necessary for the long-term stability of the restoration. Koran \& Kürschner ${ }^{(20)}$, observed an equal degree of cure, residual monomer concentration and surface hardness with both sequential and continuous light curing, as long as the total irradiation dose was adequate ${ }^{(20)}$.

Different approaches to modulate the polymerization process are in use. For instance, the soft-start technique uses reduced power density at the beginning of polymerization and slowly increases the intensity in the course of the curing process, which improves marginal adaptation $^{(46,47)}$.

A few variations of the soft-start technique were introduced; one of them is known as pulse-delay technique. The aim of this technique is to reduce the shrinkage stress by prolonging the duration of the pre-gel phase. As a result, the material maintains its plasticity for an extended period of time before it cures. During the first stage of this technique, a short curing step at reduced intensity is performed, followed by a period without exposure. Then, the final curing step is performed. This technique should reduce the formation of marginal gaps and enamel cracks ${ }^{(48,49)}$, because of the enhanced flow of the composite material.

\section{INTERMITTENT LIGHT-CURING PRESENTS ANOTHER RELATIVELY NEW TECHNIQUE(50)}

The composite is polymerized during short periods of light-on and light-off. Similar to the other techniques, the aim of this approach is to reduce the polymerization-induced stress by using light-off periods. Alonso et al. ${ }^{(51)}$, observed improved marginal adaptation in conjunction with this method.

Careful analysis is required to decide which of these techniques is most beneficial and suitable for clinical practice. A few studies reported better results for the intermittent curing technique than for other techniques ${ }^{(48,50,51,52)}$; however, higher light intensities were used for the other methods. When a low energy dose is applied, the degree of shrinkage and therefore the shrinkage stress are reduced. To avoid this problem, Cunha et al.(53), introduced a standardized energy dose of $14 \mathrm{~J} / \mathrm{cm}^{2}$. They compared the shear bond strength of samples cured with continuous curing, pulse delay, softstart and intermittent light. They did not find a significant difference among the groups of samples that were cured with methods that use continuous light. However, a tendency towards higher bond strength values was found in conjunction with low energy densities. The softstart and pulse-delay groups produced the highest bond strength values, confirming the results of other studies ${ }^{(54,55)}$.

As already shown, the main problem described in the literature is the expenditure of time required for curing composites when the softstart or pulse-delay technique is used. Cunha et al. ${ }^{(53)}$, showed that, in conjunction with the pulse-delay technique, the highest benefits can be achieved if the material is cured for 5 seconds, followed by a pause of 3 minutes and then cured for 19 seconds. If 4 or 5 increments are applied, the curing time may easily amount to 15 minutes and more. The same study showed that the soft-start technique, which involves a curing time of approximately 30 seconds, would represent a more practicable approach as the benefits of a modulated technique are in balance with a reasonable curing time. 


\section{CONCLUSIONS}

The polymerization of dental composites represents a very complex process. The dental practitioner needs to be aware of the limitations and factors affecting this process. The LED curing lights have been dramatically improved over the past few years, however they face a new challenge: they need to offer the broadband spectrum required for modern esthetic composites, which use other initiators than camphorquinone, such as Lucirin TPO 8 . Some manufactures have already begun to produce the third generation of LEDs, e.g. polywave ${ }^{\circledR}$ LED, and these curing lights have shown promising results. Furthermore, it is indispensable for LED lights to be equipped with a light guide, which should ideally be $10 \mathrm{~mm}$ wide and parallel. If the curing light produces sufficient energy, turbo light guides should be avoided to prevent an unnecessary loss of energy when the composite is cured at a distance from the light emission window. In the course of several years of research, it has gradually emerged that sophisticated curing techniques are not capable of eliminating shrinkage stress. Nonetheless, modulated techniques offer clear advantages. The soft-start techniques presents the best compromise between curing time, mechanical properties ${ }^{(56)}$, bond strength and reduction of shrinkage stress ${ }^{(57)}$.

\section{CONFLICT OF INTEREST STATEMENT}

None declared.

\section{REFERENCES}

1. Buonocore $M$. Adhesive sealing of pits and fissures for caries prevention, with use of ultraviolet light. J Am Dent Assoc, 1970; 80: 324-330.

2. Rueggeberg FA. State-of-the-art: Dental photocuring-a review. Dent Mater, 2011 Jan; 27(1): 39-52.

3. Stansbury JW. Curing dental resins and composites by photopolymerization. $J$ Esthet Dent, 2000; 12: 300-308.

4. Hofmann N, Hugo B, Schubert K, Klaiber B. Comparison between a plasma arc light source and conventional halogen curing units regarding flexural strength, modulus, and hardness of photoactivated resin composites. Clin Oral Investig, 2000; 4(3): 140-147.

5. Park SH, Krejci I, Lutz E. Microhardness of resin composites polymerized by plasma arc or conventional visible light curing. Oper Dent, 2002; 27(1): 30-37. 6. Sharkey S, Ray N, Burke F, Ziada H, Hannigan A. Surface hardness of lightactivated resin composites cured by two different visible-light sources: An in vitro study. Quintessence Int, 2001; 32(5): 401-457.

7. Fujibayashi K, Ishimaru K, Kohno A. A study on light activation units using blue light-emitting diodes. J Jpn Dent Pres Acad, 1996; 39; 1: 180-188.

8. Polydorou O, Manolaxis A, Hellwig E, Hahn P. Evaluation of the curing depth of two translucent composite materials using a halogen and two LED curing units. Clin Oral Invest, 2008; 12: 45-51.

9. Jandt KD, Mills RW, Blackwell GB, Asworth SH. Depth of cure and compressive strength of dental composites cured with blue light emitting diodes (LEDs). Dent Mater, 1999; 16: 41-47.

10. Stahl F, Ashworth SH, Jandt KD, Mills RW. Light emitting diode (LED) polymerization of dental composites: Flexural properties and polymerization potential. Biomaterials, 2000; 21: 1379-1985.

11. Meyer G, Ernst CP, Willershausen B. Decrease in power output of new lightemitting diode (LED) curing devices with increasing distance to filling surface. $J$ Adhes Dent, 2002; 4: 197-204.

12. Yoon TH, Lee YK, Lim BS, Kim CW. Degree of polymerization of resin composites by different light sources. J Oral Rehabil, 2002 Dec; 29(12): 1165-1173.

13. Mills RW, Jandt KD, Ashworth SH. Dental composite depth of cure with halogen and blue light emitting diode (LED) technology. Br Dent J, 1999; 186: 388-391.

14. Price RB, Labrie D, Rueggeberg FA, Felix CM. Irradiance differences in the violet $(405 \mathrm{~nm})$ and blue $(460 \mathrm{~nm})$ spectral ranges among dental light-curing units. $J$ Esthet Restor Dent, 2010 Dec; 22(6): 363-377.

15. Kawaguchi M, Fukushima T, Miyazaki K. The relationship between cure depth and transmission coefficient of visible-light-activated resin composites. J Dent Res, 1994; 73: 516-521.

16. Davidson CL, de Gee AJ. Light-curing units, polymerization, and clinical implications. J Adhesive Dent, 2000; 2: 167-173.

17. Leloup G, Holvoet PE, Bebeiman S, Devaux J. Raman scattering determination of the depth of cure of light-activated composites: Influence of different clinically relevant parameters. J Oral Rehabil, 2002; 29: 510-515.

18. Burtscher P. Curing of composites with an Argon laser. J Dent Res, 1991; 70 (Spec Iss): Abstract 2080.

19. Cook WD, Standish PM. Cure of resin based restorative materials. II. White light photopolymerized resins. Aust Dent J, 1983; 28: 307-311.

20. Koran $P$, Kürschner $R$. Effect of sequential versus continuous irradiation of a light-cured resin composite on shrinkage, viscosity, adhesion, and degree of polymerization. Am J Dent, 1998; 11(1): 17-22.

21. Fan PL, Schumacher RM, Azzolin K, Geary R, Eichmiller FC. Curing-light intensity and depth of cure of resin-based composites tested according to international standards. J Am Dent Assoc, 2002; 133: 429-434.

22. Rueggeberg FA, Jordan DM. Effect of light tip distance on polymerization of resin composite. Int J Prosthodont, 1993; 6: 364-370.

23. Rueggeberg FA, Cauhman WF, Curtis JW. Effect of light intensity and exposure duration on cure resin composite. Oper Dent, 1994; 19: 26-32.

24. Scott BA, Felix CA, Price RB. Effect of disposable infection control barriers on light output from dental curing lights. J Can Dent Assoc, 2004 Feb; 70(2): 105-110. 25. Ernst CP, Busemann I, Kern T, Willershausen B, Feldtest zur Lichtemissionsleistung von. Polymerisationsgeräten in zahnärztlichen. Praxen, DZZ 60, 2006; 9: 466-471.
26. McAndrew R, Lynch CD, Pavli M, Bannon A, Milward P. The effect of disposable infection control barriers and physical damage on the power output of light curing units and light curing tips. Br Dent J, $2011 \mathrm{Apr}$; 23(8): E12.

27. Mitton $\mathrm{BA}$, Wilson $\mathrm{NH}$. The use and maintenance of visible light activating units in general practice. Br Dent J, 2001 Jul 28; 191(2): 82-86.

28. Park YJ, Chae KH, Rawls HR. Development of new photoinitiation system for dental light-cure composite resin. Dent Mater, 1999; 15: 120-127.

29. Severin C, Maquin M, Husson R. Argon ion laser beam as restorative resin photocuring agent: Clinical requirement, power output specification (Abstract 73 ). $J$ Dent Res, 1986; 65: 123.

30. Neumann M, Miranda W, Schmitt C, Rueggeberg F, Correa I. Molar extinction coefficients and the photon absorption efficiency of dental photoinitiators and light curing units. J Dent, 2005; 33: 525-532.

31. BASF. Technical information of coating raw materials, June 2001. BASF Corporation Dispersions \& Paper Chemicals Charlotte Technical Center 11501 Steele Creek Road Charlotte, NC 28273.

32. Giorgi MC, Aguiar FH, Soares LE, Martin AA, Liporoni PC, Paulillo LA. Does an additional UV LED improve the degree of conversion and Knoop Hardness of lightshade composite resins? Eur J Dent, 2012 Oct; 6(4): 396-401.

33. Santini A, Miletic V, Swift MD, Bradley M. Degree of conversion and microhardness of TPO-containing resin-based composites cured by polywave and monowave LED units. J Dent, 2012 Jul; 40(7): 577-584.

34. Lutz F, Kejci I, Luescher B, Oldenburg TR. Improved proximal marginal adaptation of class II composite restorations by use of light reflecting wedges. Quintessence Int, 1986; 17: 659-664.

35. De Goes MF, Rubbi E, Baffa O, Panzeri H. Optical transmittance of reflecting wedges. Am J of Dent, 1992; 5: 78-80.

36. Lösche GM. Marginal adaptation of class II composite fillings: Guided polymerization vs reduced light intensity. J Adhes Dent, 1999 Spring; 1: 31-39.

37. Vogel K, Salz U. Influence of trans-tooth curing on physical properties of composite (Abstract 137). J Dent Res, 1997; 76: 1111.

38. Versluis A. Tanbirojn D, Douglas WH. Do dental composite always shrink toward the light? J Dent Res, 1998; 77(6): 1435-1445.

39. Hansen EK, Asmussen E. Visible light-curing units: Correlation between depth of cure and distance between exit window and resin surface. Acta Odontol Scand, 1997 55: $162-166$.

40. Pires JA, Cvitko E, Denehy GE, Swift EJ Jr. Effects of curing tip distance on light intensity and composite resin microhardness. Quintessence Int, 1993; 24: 517-521. 41. Prati C, Chersoni S, Montebugnoli L, Montanari G. Effect of air, dentin and resinbased composite thickness on light intensity reduction. Am J Dent, 1999; 12: 231 234

42. Price RB, Derand T, Sedarous M, Andreou P, Loney RW. Effect of the distance on the power density from two light guides. J Esthet Dent, 2000; 12: 320-327.

43. Price RB, Labrie D, Whalen JM, Felix CM. Effect of distance on irradiance and beam homogeneity from 4 light-emitting diode curing units. J Can Dent Assoc, 2011; 77: b9. 44. Cunha LG, Alonso RCB, Sinhoreti MAC, Goes MF, Correr-Sobrinho L. Effect of curing methods and base materials on the stress generated by the polymerization shrinkage of a resin composite. Braz J Oral Sci, 2004; 3: 609-614.

45. Feilzer AJ, Dooren LH, de Gee AJ, Davidson CL. Influence of light intensity on polymerization shrinkage and integrity of restoration-cavity-interface. Eur J Oral Sci, 1995; 103: 322-326.

46. Yoshikawa T, Burrow MF, Tagami J. A light curing method for improving marginal sealing and cavity wall adaptation on resin composite restorations. Dent Mater 2001; 17: 359-366.

47. Mehl A, Hickel R, Kunzelmann KH. Physical properties and gap formation of light cured composites with and without "soft start polymerization". J Dent, 1997; 25: 321 330

48. Kanca J, Suh BI. Pulse activation: Reducing resin-based composite contraction stresses at the enamel cavosurface margins. Am J Dent, 1999; 12: 107-112.

49. Suh BI, Feng L, Wang Y, Cripe C, Cincione F, Rjik W. The effect of pulse-delay cure technique on residual strain in composites. Compend Contin Educ Dent, 1999; 20: 4-12. 
50. Obici AC, Sinhoreti MAC, de Goes MF, Consani S, Sobrinho LC. Effect of the photo-activation method on polymerization shrinkage of restorative composites. Oper Dent, 2002; 27: 192-198.

51. Alonso RCB, Cunha LG, Correr GM et al. Association of photoactivation methods and low modulus liners on marginal adaptation of composite restorations. Acta Odontol Scand, 2004; 62: 298-304.

52. Cunha LG, Sinhorei MAC, Consani S, Sobrinho LC. Effect of different curing methods on the polymerization depth of a light-activated composite. Oper Dent 2003; 28: 155-159.

53. Cunha LG, Alonso RCB, Correr G, Cunha W, Correr-Sobrinho L, Sinhoreti MA. Effect of different photoactivation methods on the bond strength of composite resin restorations by push-out test. Quintessence international, 2008; 39(3): 243-249.

54. Alonso RC, Cunha LG, Correr GM, Brandt WC, Correr-Sobrinho L, Sinhoreti MAC. Relationship between bond strength and marginal and internal adaptation of composite restorations photocured by different methods. Acta Odontol Scand, 2006; 64: 306-313.
55. Uno S, Tanaka T, NatzuizakA, Abo T. Effect of slow-curing on cavity wall adaptation using a new intensity-changeable light source. Dent Mater, 2003; 19: 147-152.

56. Poggio C, Lombardini M, Gaviati S, Chiesa M. Evaluation of vickers hardness and depth of cure of six composite resins photo-activated with different polymerization modes. J Conserv Dent, 2012 Jul; 15(3): 237-241.

57. Feitosa VP, Fugolin AP, Correr AB, Correr-Sobrinho L, Consani S, Watson TF, Sinhoreti MA, Sauro S. Effects of different photo-polymerization protocols on resindentine $\mu$ TBS, mechanical properties and cross-link density of a nano-filled resin composite. J Dent, 2012 Oct; 40(10): 802-809. 